\title{
Two-quantum-path interferences in high order harmonic generation
}

\author{
A. Zaïr, M. Holler, A. Guandalini, F. Schapper, J. Biegert and U. Keller \\ Physics Department,ETH, CH-8093 Zürich, Switzerland \\ e-mail: zair@phys.ethz.ch \\ P. Salières and T. Auguste \\ Service des Photons, Atoms, et Molécules, Centre d'études de Saclay, 91191 Gif-sur-Yvette, France \\ E. Cormier \\ Centre Laser Intense et Application, Université Bordeaux I, UMR 5107, 33405 Talence cedex
}

\author{
A. Wyatt, A. Monmayrant and I. Walmsley \\ Clarendon Laboratory, Parks Road, Oxford OXI 3PU, UK
}

\begin{abstract}
We have investigated intensity dependent high-harmonic generation, when short and long trajectories are both visible in the generated signal. We have measured a plateau-harmonic spectral broadening and yield modulations consistent with quantum-paths interferences calculations.

C2007 Optical Society of America
\end{abstract}

OCIS codes: (020.0020) Atomic and molecular physics; (320.7120) Ultrafast phenomena

To understand the high-order-harmonic generation process and especially how it can result in the generation of attosecond pulse trains, researchers have developed a quantum-mechanical theory to investigate quantum-paths effects [1]. These quantum-paths correspond physically to trajectories that an electron follows in the generation process: emission into the continuum by tunnel ionization, acceleration in the laser field and recombination with emission of a harmonic photon. Over all the possible classes of quantum-paths, it was shown that mainly two classes contribute significantly to the generation of plateau harmonics, referred to as "short" and "long" trajectories and that those two classes merge in the cutoff region. Experimental studies showed the possibility of selecting one of the two trajectories in the macroscopic response thanks to phase matching during propagation [2]. In that case, single atom calculations have shown that for each individual trajectory, the dipole response does not show any modulation with respect to the fundamental laser intensity. However, if the two trajectories co-exist, intensity dependent modulations are expected because of the different dipole phases of the long and short trajectory. Here, we present the first experimental observation that can be related to these quantum-path interferences.

Our experiments are based on a laser system, with high stability, delivering 30 -fs infrared (IR) pulses at a repetition rate of $1 \mathrm{kHz}$ with maximum pulse energy of $1.5 \mathrm{~mJ}$. This laser beam enters a vacuum chamber and is focused by a 500-mm (ROC) spherical mirror into a pulsed gas jet of Argon. A variable attenuator is placed in the IR beam to control finely the peak intensity in the jet from $0.5 \times 10^{14} \mathrm{~W} / \mathrm{cm}^{2}$ to $2.5 \times 10^{14} \mathrm{~W} / \mathrm{cm}^{2}$. In Fig. 1 , the harmonic spectra as a function of laser peak intensity are shown. In the upper part, the jet was placed after the laser focus position to select the short trajectory only. As expected, the harmonics are narrow and their amplitudes saturate monotonically with the laser intensity. In the lower part, the jet was positioned before the laser focus. Far- 


\section{JFC6.pdf}

field spatial filtering was used before the XUV spectrometer to select comparable contributions of the two quantum paths, making use of their different divergence. The figure shows a clear broadening of the plateau harmonic spectral width (from order 13 to 25) consistent with the long trajectory contribution [2]. Indeed, the latter presents a large frequency chirp due to a much faster intensity dependence of the harmonic dipole than for the short path contribution. By increasing the laser peak intensity, the chirp is increased, broadening the harmonics but also separating spectrally the long and the short trajectory signatures The amplitude of the plateau-harmonics is clearly modulated with increasing laser peak intensity (Fig. 2). This particular intensity dependence is visible all over the plateau region. However, for the cutoff harmonics, as expected, no modulation or broadening is observed since the two trajectories have merged in a single one.

In order to compare the observed interferences with predictions from theory, SFA and TDSE calculations were performed. Figure 2 presents the experimental amplitude modulations of harmonic 19, spectrally integrated over one harmonic order, and the dipole amplitudes from single atom response obtained by SFA and TDSE calculations. As can be seen, the first few experimentally observed modulations are consistent with the theoretical computations. This indicates that the experimentally measured modulations are indeed due to the coexistence of the two trajectories. To our knowledge, this is the first experimental observation consistent with two-quantum-path interferences predicted by theory more than a decade ago.
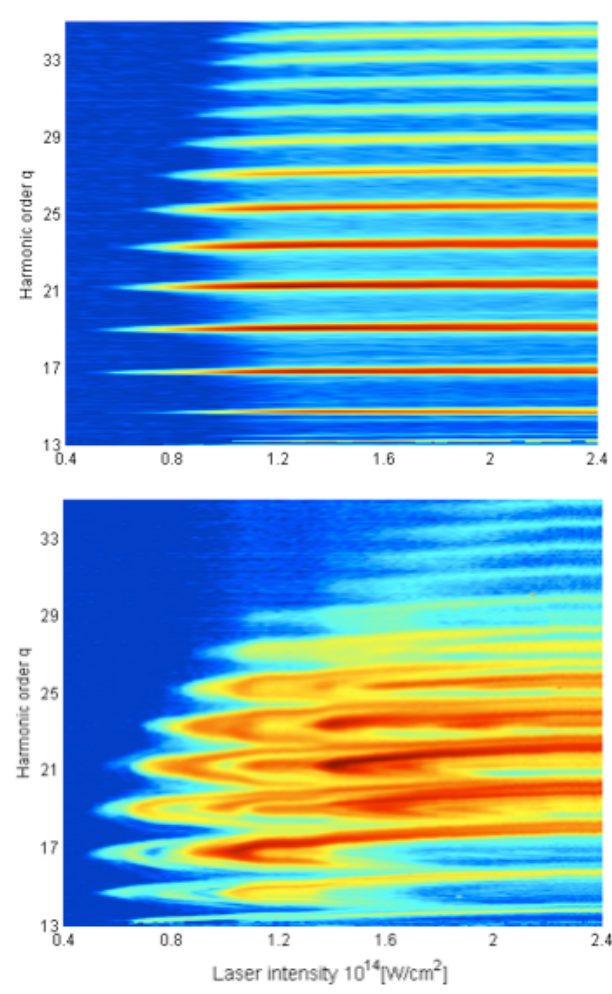

Figure 1: Harmonic spectra as a function of the laser peak intensity: In the upper part, the jet was placed after the laser focus position (short trajectory). In the lower part, the jet was positioned before the laser focus (short and long trajectories).

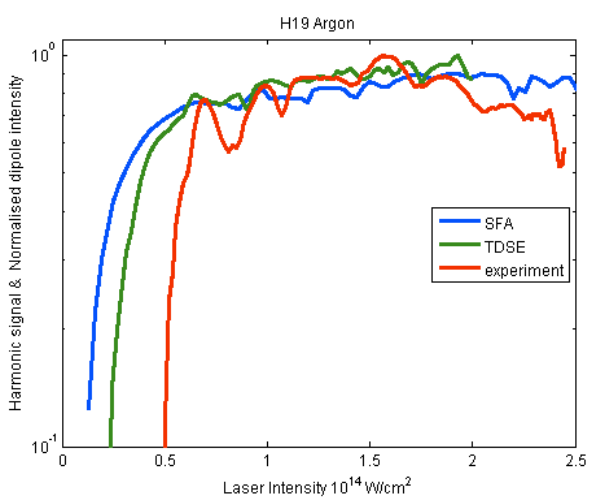

Figure 2: $19^{\text {th }}$ experimental harmonic amplitude normalized and spectrally integrated between order 18 to 20 (red curve), and $19^{\text {th }}$ single atom harmonic intensity obtained with the SFA (blue curve) and the TDSE (green curve) theoretical model as a function of the laser peak intensity.

[1] P. Antoine et al., PRL 77, 1234, (1996).

[2] G. Sansone et al., PRA 73, 053408 (2006). 